\title{
Bistable polarization switching in mutually coupled vertical-cavity surface-emitting lasers
}

\author{
Raúl Vicente, Josep Mulet, and Claudio R. Mirasso \\ Departamento de Física Interdisciplinar, IMEDEA, CSIC-UIB, E-07122, Palma de Mallorca, Spain \\ Marc Sciamanna \\ Supélec, LMOPS CNRS UMR-7132, 2 Rue Edouard Belin, F-57070, Metz, France
}

\begin{abstract}
We theoretically investigate the polarization-resolved dynamics of two vertical-cavity surface-emitting semiconductor lasers that are mutually coupled through coherent optical injection. We find a sequence of bistable polarization switchings that can be induced by either changing the coupling strength or the optical propagation phase. The successive polarization switchings are correlated to the creation of new compound-cavity modes when these parameters are continuously varied. (c) 2005 Optical Society of America
\end{abstract}

OCIS codes: $250.5270,260.5430$

The understanding and control of the polarization degree of freedom in lasers is of fundamental importance in any polarization sensitive application. The heterostructure of a conventional edge-emitting laser induces a large anisotropy between TE and TM modes. Therefore, these devices generally emit in a single and well defined polarization state, unless induced strain or other band structure engineering techniques are applied. On the other hand, vertical-cavity surface-emitting lasers (VCSELs) preferentially emit linearly polarized (LP) light along one of two orthogonal preferred directions $(\hat{x}$ and $\hat{y})$ due to the weak material and cavity anisotropies. However, polarization switching between $\hat{x}$ and $\hat{y}$ is often observed when either varying the temperature and/or the injection current,, when feeding back part of the emitted light,, or when injecting external light $!^{[3}$ In mutually coupled edge-emitting lasers, achronal synchronization of chaotic coupling-induced instabilities, symmetry-breaking,,$^{4}$ and the role of asymmetries ${ }^{[5}$ have been reported. Recent experimental studies ${ }^{6}$ have demonstrated that the mutual coupling of two similar VCSELs can also induce instabilities with high synchronization degree in both total intensity and polarization.

In this letter, we investigate the mutual coupling of two similar VCSELs in order to determine the role of light polarization dynamics in their mutual entrainment. In this configuration, we find a coupling-induced 
polarization switching (PS) scenario, where multiple PS occur when continuously varying either the coupling strength or the propagation phase between the two lasers. Each PS event is correlated to the creation of a new linearly polarized compound-cavity mode with higher gain. In addition, a bistable region appears around each PS which hysteresis width can be controlled by varying the coupling parameters. Controllable bistable PS in mutually-coupled VCSELs may add new functionalities to those applications employing a bistable region for fast switching applications. ${ }^{[]^{7}}$

Each solitary VCSEL is described according to the spin-flip model ${ }^{[8}$ For moderate coupling strength, the interaction between the lasers is taken into account by including delayed optical injection terms. $\frac{9}{9}$ The equations governing each laser read

$$
\begin{aligned}
\dot{E}_{1,2 \pm}= & \kappa(1+i \alpha)\left[N_{1,2} \pm n_{1,2}-1\right] E_{1,2 \pm} \\
& -\left(\gamma_{a}+i \gamma_{p}\right) E_{1,2 \mp}+\xi e^{-i \Omega \tau} E_{2,1 \pm}(t-\tau) \\
& +F_{1,2 \pm}(t) \\
\dot{N}_{1,2}= & -\gamma_{e}\left[N_{1,2}-\mu+\left(N_{1,2}+n_{1,2}\right)\left|E_{1,2+}\right|^{2}\right. \\
& \left.+\left(N_{1,2}-n_{1,2}\right)\left|E_{1,2-}\right|^{2}\right], \\
\dot{n}_{1,2}= & -\gamma_{s} n_{1,2}-\gamma_{e}\left[\left(N_{1,2}+n_{1,2}\right)\left|E_{1,2+}\right|^{2}\right. \\
& \left.-\left(N_{1,2}-n_{1,2}\right)\left|E_{1,2-}\right|^{2}\right],
\end{aligned}
$$

where the subindices 1,2 label the lasers. $E_{ \pm}$are the circularly-polarized components of the electric field $\left(E_{ \pm}=\left(E_{x} \pm i E_{y}\right) / \sqrt{2}\right) . N$ represents the total inversion population while $n$ is the difference of population inversions between the up/down spin reservoirs associated to emission of opposite circularly-polarized photons. We consider the same internal parameters for both VCSELs and a perfect alignment between their two $\hat{x}$ and $\hat{y}$ eigenaxes. The meaning and values of the parameters in Eqs. (11)-(3) are: linewidth enhancement factor $\alpha=3$, cavity decay rate $\kappa=300 \mathrm{~ns}^{-1}$, total carrier number decay rate $\gamma_{e}=1 \mathrm{~ns}^{-1}$, amplitude anisotropy $\gamma_{a}=-0.1 \mathrm{~ns}^{-1}$, phase anisotropy $\gamma_{p}=3 \mathrm{~ns}^{-1}$. We fix the normalized pump at $\mu=1.5$, where the solitary VCSELs emits in a stable $\hat{x}$-polarization. The spin-flip rate is $\gamma_{s}=1000 \mathrm{~ns}^{-1}$, as reported in experiments on PS in VCSELs $\frac{10]}{10}$ The distance between both VCSELs is only $L=6 \mathrm{~cm}$ (coupling time $\tau=0.2 \mathrm{~ns}$ ). $\Omega$ is the optical frequency of the two free-running lasers. The coupling strength $\xi$ and propagation phase $\Omega \tau$ are our bifurcation parameters. The last term in the field Eq. (1) are Langevin noise sources that account for spontaneous emission processes $F_{ \pm}(t)=\sqrt{\beta \gamma_{e}(N \pm n)} \chi_{ \pm}(t)$, where $\chi_{ \pm}(t)$ are independent complex random numbers with zero mean and $\delta$-correlation. The spontaneous emission factor is $\beta=10^{-5}$.

Fig. 1 1 shows maps of the LP mode intensities of laser 1 upon variation of the coupling strength $(\xi)$ and 
propagation phase $(\Omega \tau \bmod 2 \pi)$. Intensities are plotted after removing transients and averaging over $50 \mathrm{~ns}$. The alternation between dark and light regions demonstrates successive PS between orthogonal LP states. Moreover, PS events appear with a clear periodicity in the coupling parameters. The maps of laser 2 are not shown in the figure because PS occur for the same coupling conditions in both VCSELs. Interestingly, numerical simulations show that the coupling-induced PS scenario is robust against small mismatch of the laser parameters: linear anisotropies, detuning and misalignment of polarization axes.

In order to gain insight into the origin of the coupling-induced PS, we study the bifurcations of LP solutions of Eqs. (1)-(3) as the coupling strength and phase are changed. Two kinds of monochromatic LP solutions appear. Symmetric (asymmetric) fixed points corresponds to identical (different) output power and inversion of both lasers. In our case, numerical simulations indicate that only symmetric LP solutions are stable. Therefore, we concentrate our analysis to the symmetric fixed points. They are obtained by imposing the steady-state conditions $E_{1+}=E_{0} e^{i \omega t}, E_{1-}=E_{0} e^{i(\omega t+\varphi)}, E_{2+}=E_{0} e^{i(\omega t+\phi)}, E_{2-}=E_{0} e^{i(\omega t+\phi+\varphi)}$, $N_{1,2}=N_{0}$, and $n_{1,2}=0$, where $\varphi$ controls the polarization direction and $\phi$ takes into account the relative phase between the electric fields of both lasers. These conditions are only satisfied for a relative phase $\phi=0$ $(\phi=\pi)$ leading to in-phase (anti-phase) solutions. After a little of algebra, the frequency shift and inversion of the symmetric monochromatic solutions read

$$
\begin{aligned}
\omega & = \pm\left(\alpha \gamma_{a}-\gamma_{p}\right) \\
& -\xi \sqrt{1+\alpha^{2}} \sin (\phi-\Omega \tau-\omega \tau-\arctan \alpha), \\
N & =\frac{1}{\kappa}\left[\kappa \pm \gamma_{a}-\xi \cos (\phi-\Omega \tau-\omega \tau)\right],
\end{aligned}
$$

where \pm stands for $\hat{x}$ and $\hat{y}$ states. As shown in Fig. 2 the corresponding LP steady-states along the $\hat{x}$ and $\hat{y}$ polarization directions are located on two different ellipses in the frequency $(\omega)$ versus inversion $(N)$ phase space. The steady-states are plotted for increasing values of the coupling strength near the transition to a PS event $\left(13 \leq \xi \leq 13.8 \mathrm{~ns}^{-1}\right)$. For $\xi=13 \mathrm{~ns}^{-1}$ in panel (a), the system operates in the lowest inversion fixed point, which in this case corresponds to an in-phase $\hat{y}$-LP solution. An increase of the coupling strength in panel (b) creates a new pair of $\hat{x}$-LP modes through a saddle-node bifurcation. One of these two modes is a stable node, hence accessible as a stable attractor for the laser system. However, at this stage the system still operates in the most stable maximum gain mode (MGM) of the $\hat{y}$-polarized ellipse. For larger coupling strengths [panel (c)], the $\hat{y}$-LP MGM destabilizes to a limit cycle through a Hopf bifurcation at the relaxation oscillation frequency. Further increasing $\xi$, the oscillatory dynamics is interrupted and the laser finally switches to the $\hat{x}$-LP in-phase fixed point, which has become the new MGM [panel (d)]. If the 
coupling rate is continously increased this process repeats and new PS are periodically induced following the same mechanism. When the procedure is repeated decreasing the coupling from large values, bistability is observed since the saddle-node bifurcation, that creates the stable x-LP mode, is located at a smaller $\xi$ than the PS point. Numerical simulations show that the coupling-induced PS scenario is qualitatively preserved for different $\gamma_{s}$ values ranging from $50 \mathbf{n s}^{-1}$ to $10^{4} \mathbf{n s}^{-1}$. However, the range of coupling strengths around PS where total intensity instability appears increases for small $\gamma_{s}$. Moreover, the amount of spontaneous emission noise slightly modifies the PS positions. Since two stable orthogonal LP attractors coexist around each PS, noise may favor the jump to an orthogonal LP.

From the simulations, we find that the periodicity of PS, as $\xi$ is varied, approximately equals the periodicity in the creation of a new saddle-node pair. It can be demonstrated that, for a fixed polarization and relative phase, the number of symmetric steady-states of Eq. (44)-(洍) is proportional to $1+\xi \tau\left(1+\alpha^{2}\right)^{1 / 2} / \pi$. Consequently, taking into account that the creation of new steady-states alternates between in-phase and anti-phase modes, the periodicity in the PS events when $\xi$ is changed in a definite direction is approximately $\Delta \xi_{P S}=\pi / \tau\left(1+\alpha^{2}\right)^{1 / 2}$. This value corresponds to the increase in $\xi$ necessary to create a new pair of modes with a given polarization. For our set of parameters this quantity corresponds to $4.96 \mathrm{~ns}^{-1}$, which agrees

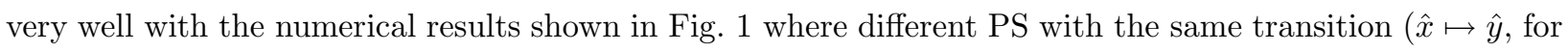
example) are observed to be separated by $\sim 5 \mathrm{~ns}^{-1}$.

The PS events induced by changing the propagation phase can also be understood in terms of the bifurcation of the LP solutions. When the phase is continously decreased from $2 \pi$ to 0 there is a pulling of the steady-states around the ellipses from the low to high inversion regions. At the same time, a new pair of saddle-node modes is created at the lowest vertex of each of the ellipses while they are annihilated by an inverse saddle-node bifurcation at the highest vertex. Since the process of creation of new pairs of modes at the lowest corner of the ellipse occurs in alternation for the $\hat{x}$ and $\hat{y}$ polarization modes, this results into PS events when varying the propagation phase. The transformation $\Omega \tau \mapsto \Omega \tau+\pi$ interchanges the in-phase and anti-phase modes and defines the periodicity of the PS induced by phase changes. Similar bifurcation mechanism has been reported in mutually coupled edge-emitting lasers.11

In our VCSEL system the selection of stable compound-cavity modes may be accompanied by new features such as polarization switching with hysteresis. Fig. 3 shows the multiple PS events when varying $\xi$ or $\Omega \tau$, clarifying the bistability that occurs when increasing and then decreasing the control parameter. When sweeping the coupling strength both the switch-off and switch-on events of the x-LP mode are accompanied by hysteresis, whose widths are labelled as $H_{1}$ and $H_{2}$ respectively (a). The orthogonal polarization component (not shown) displays a complementary behavior. Interestingly, as shown in (c), $H_{1}$ and $H_{2}$ grow while increasing $\xi$, hence showing the hysteresis width can be tuned with the coupling parameters. A scan of 
the propagation phase also leads to multiple PS (b) but, in contrast to previous case, i) only the switch-on events of the x-LP mode are accompanied by bistability, and ii) the hysteresis width keeps constant when changing $\Omega \tau$ as a consequence of the symmetry of Eqs. (10)-(3) with respect to a change of $\pi$ in $\Omega \tau$.

In conclusion, we have shown that mutually coupled VCSELs may lead to robust multiple bistable PS. A sequence of PS events appear when varying the coupling strength or the propagation phase, with a periodicity that is related to the creation of new compound-cavity modes with higher gain and orthogonal polarization. Each PS event is accompanied by a large hysteresis whose width can be tuned by the coupling parameters. Such controllable bistable PS system is interesting for fast optical switching applications.

The authors acknowledge financial support from MEC (Spain) and Feder, project FIS2004-00953. JM is supported by the CSIC (Spain) through the program I3P-PC2003. MS acknowledges support from UIB (Spain).

\section{References}

1. K.D. Choquette, R.P. Scheneider, L.K. Lear and R.E. Liebenguth, IEEE J. Sel. Topics in Quantum Electron. 1, 661, (1995).

2. M. Sciamanna et al., Opt. Lett. 28, 1543, (2003).

3. Z. G. Pan et al., Appl. Phys. Lett. 63, 2999 (1993).

4. T. Heil, I. Fischer, W. Elsasser, J. Mulet and C.Mirasso, Phys. Rev. Lett. 86, 795 , (2001).

5. H.J. Wünsche et al., Phys. Rev. Lett. 94, 163901, (2005).

6. N. Fujiwara, Y. Takiguchi, and J. Ohtsubo, Opt. Lett. 28, 1677, (2003).

7. K. Panajotov et al., IEEE Photon. Technol. Lett. 11, 985 (1999).

8. M. San Miguel, Q. Feng, and J.V. Moloney, Phys. Rev. A 52, 1728, (1995).

9. J. Mulet, C. Masoller, and C. R. Mirasso, Phys. Rev. A 65, 063815, (2002).

10. R. F. M. Hendriks, M. P. Van Exter, J. P. Woerdman, K. H. Gulden, and M. Moser, IEEE J. Quantum Electron. 34, 1455 (1998).

11. H. Erzgraber, et al., Opt. Commun., 255, 285, (2005). 


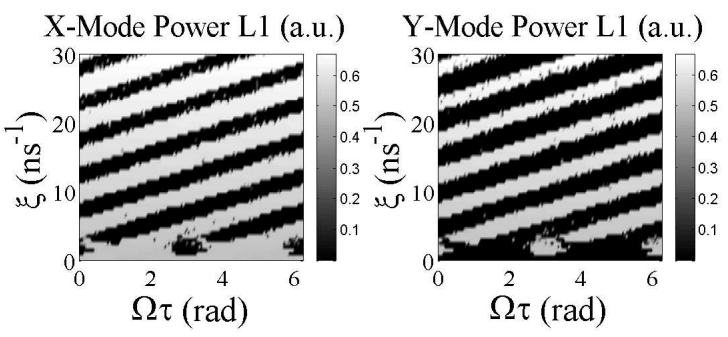

Fig. 1. Averaged intensities in the $\hat{x}$ - and $\hat{y}$-LP modes of one VCSEL as a function of $\xi$ and $\Omega \tau$. Parameters are specified in the text. 

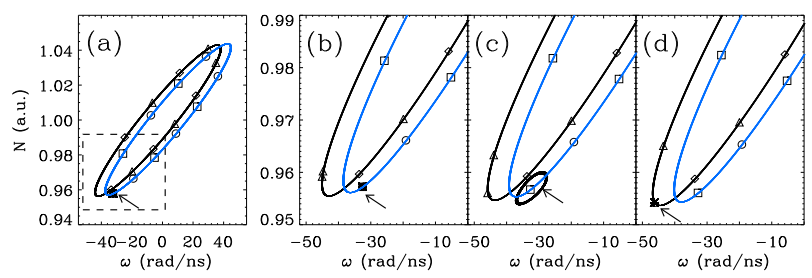

Fig. 2. Panels (a)-(d) show the location of the symmetric fixed points in the $N$ vs. $\omega$ phase space for $\xi=13$ $\mathrm{ns}^{-1}, \xi=13.25 \mathrm{~ns}^{-1}, \xi=13.6$, and $\xi=13.8 \mathrm{~ns}^{-1}$, respectively. Diamonds and triangles stand for the in-phase and anti-phase $\hat{x}$-polarized solutions. Squares and circles stand for the in-phase and anti-phase $\hat{y}$-polarized solutions. Arrows identify the attractor at which the system operates. 

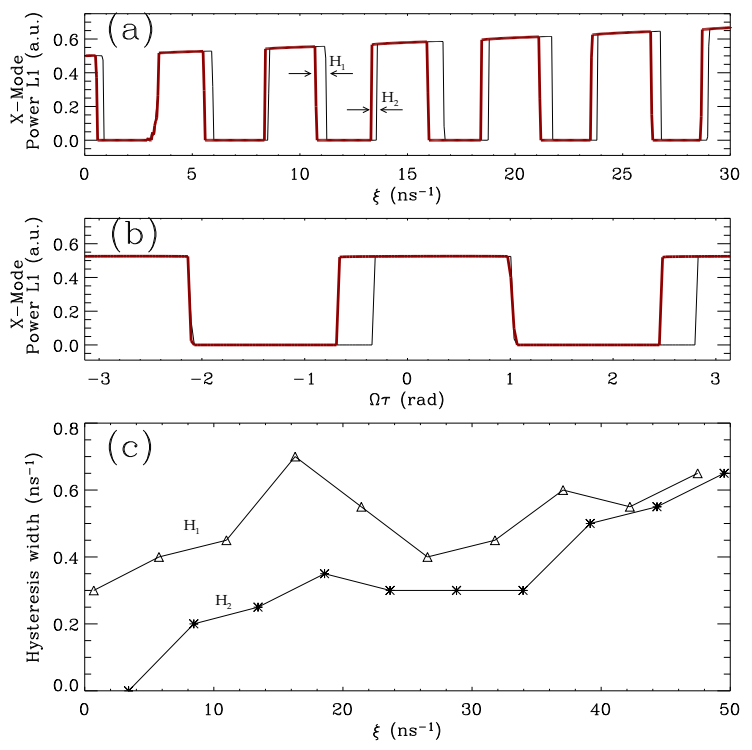

Fig. 3. Panels (a) and (b) show the evolution of the x-LP mode intensity as we increase (thin line) and then decrease (thick line) $\xi$ (a) and $\Omega \tau$ (b). In (c) are shown the two hysteresis widths $H_{1}$ and $H_{2}$ [labelled in (a)] as a function of $\xi$. 